\title{
Design of adaptive structures by kinematic synthesis of mechanisms
}

\author{
Y. Akgün \\ Gediz University Department of Interior Architecture, Izmir, Turkey \\ F. Maden \& K. Korkmaz \\ Izmir Institute of Technology Department of Architecture, Izmir, Turkey
}

\begin{abstract}
Parallel to the development of material science and computer technologies, adaptive structures and smart building components have become the demand of contemporary architecture. However, in order to design adaptive systems, basic knowledge on kinematic synthesis is needed. This paper introduces the fundamental principles of the kinematic synthesis of mechanisms and expresses the potential structural uses of common mechanisms. For this purpose, main concepts of the mechanisms are introduced and types of kinematic synthesis are explored by the help of innovative examples. In this context, firstly, type synthesis methods are explained. Second step covers the path generation rules, and the final step includes the design of multi-loop and chain mechanisms. During the explanation of the aforementioned principles, innovative adaptive structures which are designed by the authors are introduced as well. These structures are all derived from common planar and spatial mechanisms such as four-bar linkages, Watt mechanisms, scissor systems, Bennett linkages and different multi-loop mechanisms.
\end{abstract}

\section{INTRODUCTION}

For a few decades, by the development of technology and change of life styles, concepts of flexibility, mobility and adaptability have gained importance in all parts of our lives. In order to catch the demands of the era, the fields of architecture and structural engineering have been evolved and many buildings with adaptive structures, walls, façade systems and furniture have been designed. In order to design these adaptive elements and structures, an interdisciplinary approach between architecture, mechanical engineering and structural engineering is needed. However, conventional architecture and structural engineering educations do not focus on this interdisciplinary area. This paper emphasizes this deficiency and aims to expose how expertise on mechanisms could contribute to an architect; how an architect could inspire from common mechanisms while designing an adaptive system; and what kind of design principles mechanisms have.

In order to answer the aforementioned research questions, this paper begins with the introduction of the main concepts on theory of mechanisms and continues with the methods on kinematic synthesis of mechanisms. Kinematic synthesis can be defined as the systematic design of mechanisms for a given performance (Erdman \& Sandor, 1991). This systematic design approach covers the typological and dimensional design of the mechanism, path design and correlation between inputs and outputs, velocity and acceleration. This paper, firstly introduces the path design and generation from an architectural point of view. Secondly, design of a mechanism which can convert on a defined path is introduced. Finally, synthesis of multi-loop mechanisms derived from a unit mechanism is explored analytically. 


\section{MAIN TERMS}

In order to comprehend the geometric and kinematic characteristics of mechanisms, first, the concept of mechanism should be understood. A mechanism can be defined as a group of rigid bodies connected to each other by rigid kinematic pairs to transmit force and motion (Söylemez, 2009). Most movable, foldable, deployable and convertible structures behave as mechanisms during their conversion process and as a load resisting entity when they are fixed. Thus, they have the characteristics of both a mechanism and a bearing structure. These types of mechanisms are also called as structural mechanisms (Chen, 2003).

The other important concept for mechanisms is the Mobility (M). A mechanical system's mobility can be classified according to the number of degrees of freedom (DoF) that it possesses. The system's DoF is equal to the number of independent parameters that are needed to uniquely define its position in space at any instant of time (Norton, 2003). As another definition, mobility is equal to the number of additional actuators or motors needed to control or fix the system. For all transformable and convertible structures, mobility of the system is larger than or equal to one $(M \geq 1)$, and transformation capability increases according to the increase of the mobility value. If the mobility of a kinematic system is equal to zero $(\mathrm{M}=0)$, the structure is stable and requires no additional stabilization (Akgün et al, 2011). According to simplified version of Freudenstein-Alizade (1975) formula, the mobility of a system can be calculated as follows:

$$
M=J_{T}-\lambda L
$$

where $J_{T}$ includes the contribution of DoFs of all joints, $L$ is the number of loops, $\lambda$ is the number of active DoFs of the space where the mechanism operates ( $\lambda=3$ for planar systems and $\lambda=6$ for spatial systems).

\section{KINEMATIC SYNTHESIS OF MECHANISMS \& ARCHITECTURAL APPLICATIONS}

Kinematic synthesis is a systematic approach which covers the selection of suitable mechanism type according to the given path, calculation of the necessary dimensions, force and velocity of the mechanism and its elements, active workspace and form generation capacity. At this step, an introduction to the different methods of kinematic synthesis is made.

\subsection{Type Synthesis}

Type synthesis aims to determine the most suitable mechanism which meets the defined motion; and use this mechanism with small dimensional modifications. This method is the simplest and the most common way for designing adaptive structures and architectural elements. Type synthesis can be used in two different approaches: First approach covers the search of literature for the most suitable mechanism in order to meet the defined motion path. Second approach covers the search for suitable architectural or structural uses for a given mechanism. In this paper, examples of type synthesis are explained by using four-bar linkage (mechanism) which is one of the most common planar mechanisms.

A four-bar linkage (mechanism) is the simplest movable closed chain linkage. It consists of four bodies, called bars or links, connected in a loop by four joints (Figure 1a). Generally, the joints are configured so the links move in parallel planes, and the assembly is called a planar four-bar linkage (Hartenberg \& Denavit, 1964). According to Equation $1, J_{T}=4, \lambda=3, \mathrm{~L}=1$, so the mobility (M) of the four-bar linkage is equal to 1 . This means, in order to transform the four-bar linkage, at least one actuator should be necessary.

Four-bar linkage is very common in our daily life. For example, suspension systems of bicycles and automobiles, excavator diggers, some small hand tools, swing of cradles are obtained from four-bar linkages. In Figure 1b, schematic diagram of a four-bar linkage as a desk lamp can be seen. In this mechanism, a parallelogram is obtained by the equal dimensions of the input and output links. By this way, the intermediate link stays always parallel and the lamp perpendicular to the ground. 
a

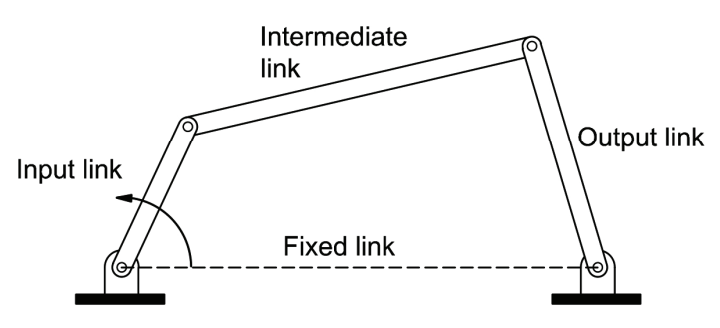

b

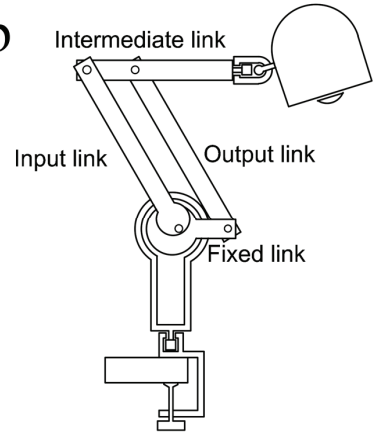

Figure 1. Four-bar Mechanism (a), Four-bar Desk Lamp Mechanism (b)

Santiago Calatrava has benefited from four-bar linkages at some of his designs. Adaptive sun shade structure of Emergency Call Center in St. Gallen-Switzerland is a good example (Figure 2). This structure is composed of several parallel four-bar linkages which are connected by an arc shaped common input link. By this connection detail, all individual four-bars move together as one mechanism.

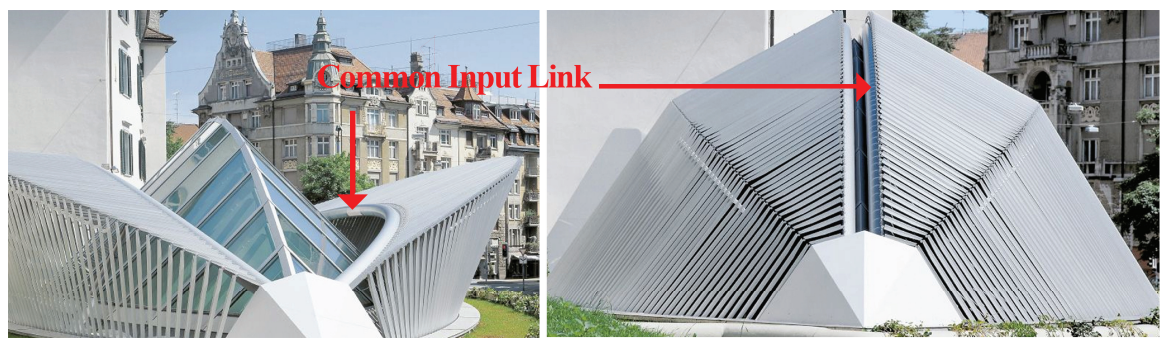

Figure 2. Emergency Call Center in St. Gallen by Santiago Calatrava (Calatrava, 2012)

Another architectural example for the use of four-bar linkage is the Sustainable Kinetic Roof Design of Yenal Akgün (Figure 3). In this project, four-bar linkage is used as the main structure of a transformable roof. By the transformation of four-bar linkage, this project proposes various green terrace alternatives for the occupants.
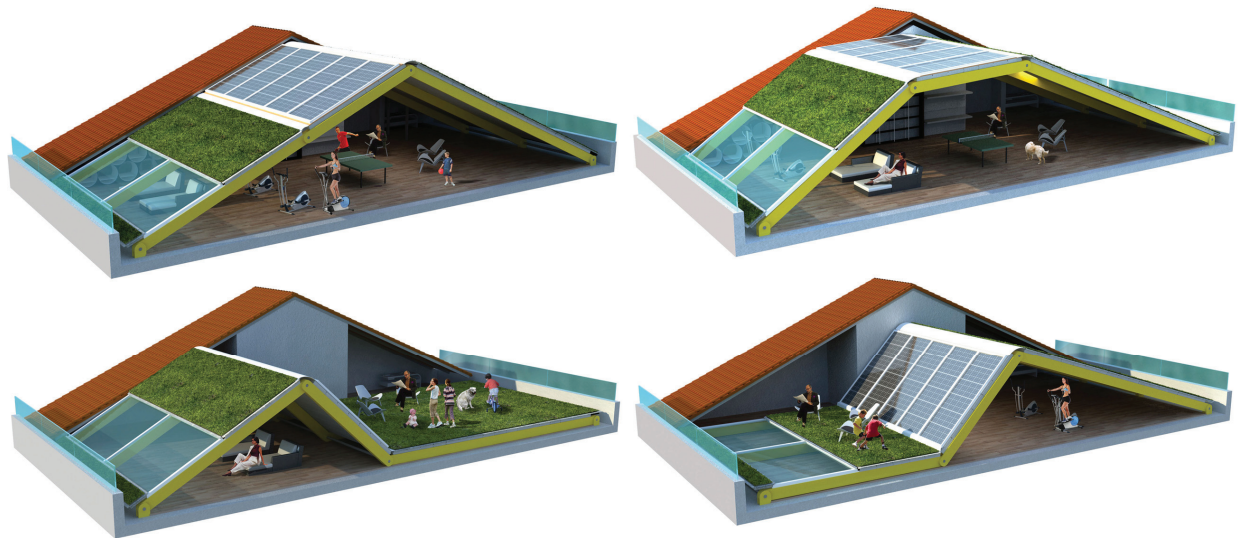

Figure 3. Sustainable Kinetic Roof Project by Yenal Akgün

Bennett linkage (mechanism) is a spatial version of four-bar linkage with hinged joints that have their axes angled in a particular way that makes the system movable (McCarthy \& Soh, 2010) 
(Figure 4a). Koray Korkmaz et al. (2012) has developed a transformable mechanism with eight planar hinges derived from Bennett linkage and has adapted this mechanism to a transformable shade design. Different from Bennett linkage, this mechanism can achieve asymmetrical transformations (Figure $4 b-c)$.
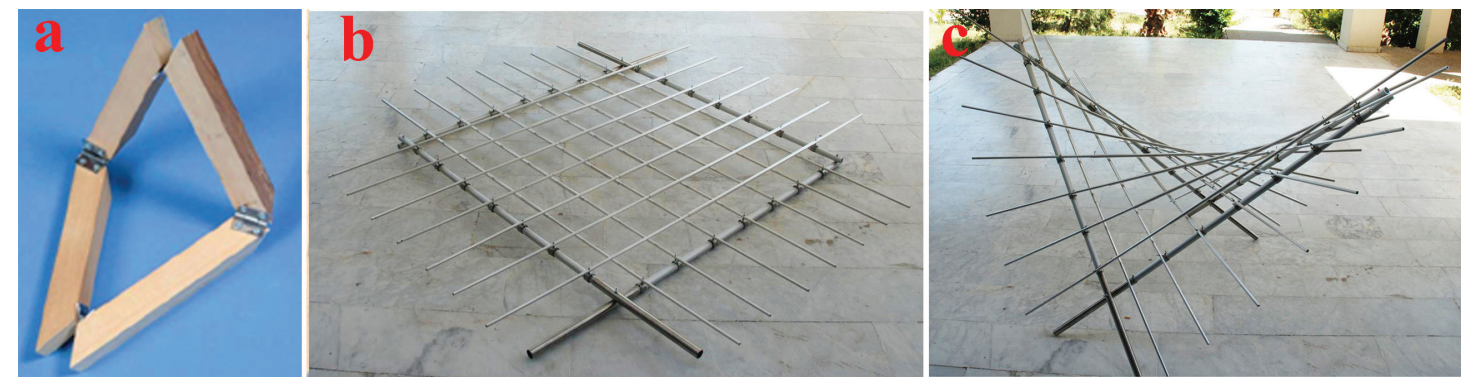

Figure 4. Bennett Mechanism and Its Application to the Transformable Shade Design by Korkmaz et al (2012)

Beside their use as adaptive roof structures, four-bar linkage have capacity to be used as various structural and architectural elements. However, in order to design such adaptive structures, designers should be familiar to the types of various mechanisms and their transformation capacities.

\subsection{Path Generation}

When defined motion path of a tracer point on a mechanism is an important design criterion, type synthesis cannot be enough for solving the problem. Dimensioning of the selected mechanism, which can meet the defined path, should be made as well. Path generation methods meet this problem.

Synthesis of a mechanism which can meet a defined motion path is a common problem not only in mechanical engineering but also in architecture, automotive industry, industrial design etc. For example, design and dimensioning of a robot arm which moves the loads from two defined points, a foldable roof structure which transforms on a designed path and foldable roof mechanism of an automobile are typical examples for path generation problem.
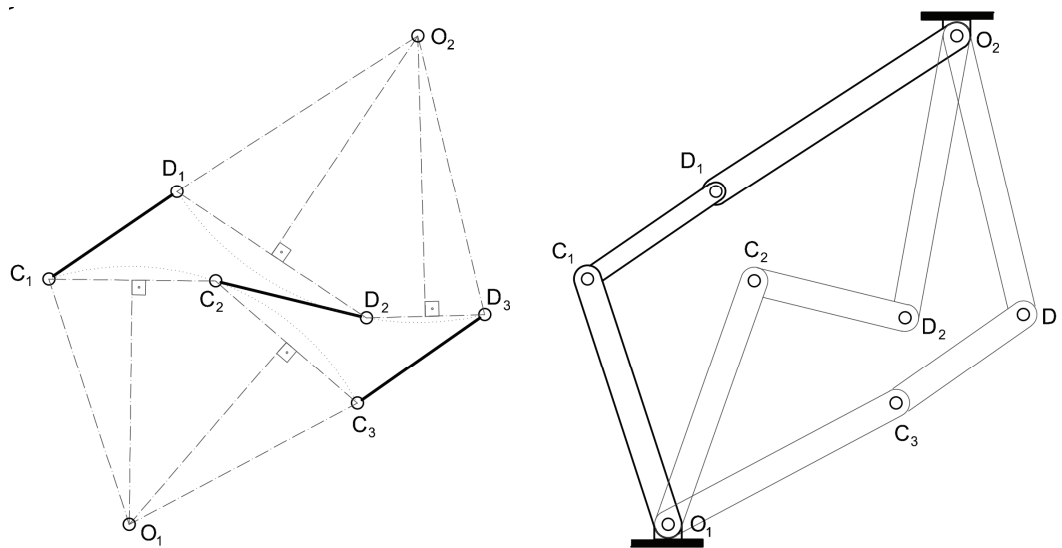

Figure 5. Path Generation of a Four-bar Linkage with Graphical Methods

Path generation problem can be solved both with graphical and analytical methods. Path generation problem of simple mechanisms with less number of links, such as four-bar linkage, can be solved easier by graphical methods. However, for complicated mechanisms with numerous links, analytical methods are more useful. In this paper, graphical solution of a four-bar has been explained. In Figure 5, three defined positions of intermediate link of a four-bar linkage are giv- 
en. According to these positions, dimensions and locations of input and output links can be found by using geometrical relations. Point $\mathrm{C}$ connects the intermediate link with input link and Point $\mathrm{D}$ with the output link. Thus, $\mathrm{C}_{1}, \mathrm{C}_{2}$ and $\mathrm{C}_{3}$ points represent the transformation of input link. According to this, the fixed point of input link should be on the center point of the circle which is tangent to points $C_{1}, C_{2}$ and $C_{3}$. The same rule can be applied to the points $D_{1}, D_{2}$ and $\mathrm{D}_{3}$ in order to find the fixed point of the output link.

With their numerous numbers of repetitive links, scissor systems are one of the most common mechanisms and Yenal Akgün and Feray Maden's studies on scissor systems can be good architectural examples for path generation. In Figure 6a, a motion path has been given and design of a scissor mechanism which can meet this path has been asked. This path is composed of two tangent arcs. It is known that for the scissor mechanisms which are derived from the multiplication of the polar scissor units, axes connecting the hinge points intersect at one point (Figure 6c) (Akgün et al, 2010). Thus, according to the given path and $l_{1}$ or $l_{2}$ dimensions, all dimensions of a scissor mechanism which can meet the path can be found by analytical or graphical methods. Analytical path generation of scissor systems has been thoroughly investigated in Maden et al (2011) and Akgün et al. (2007).

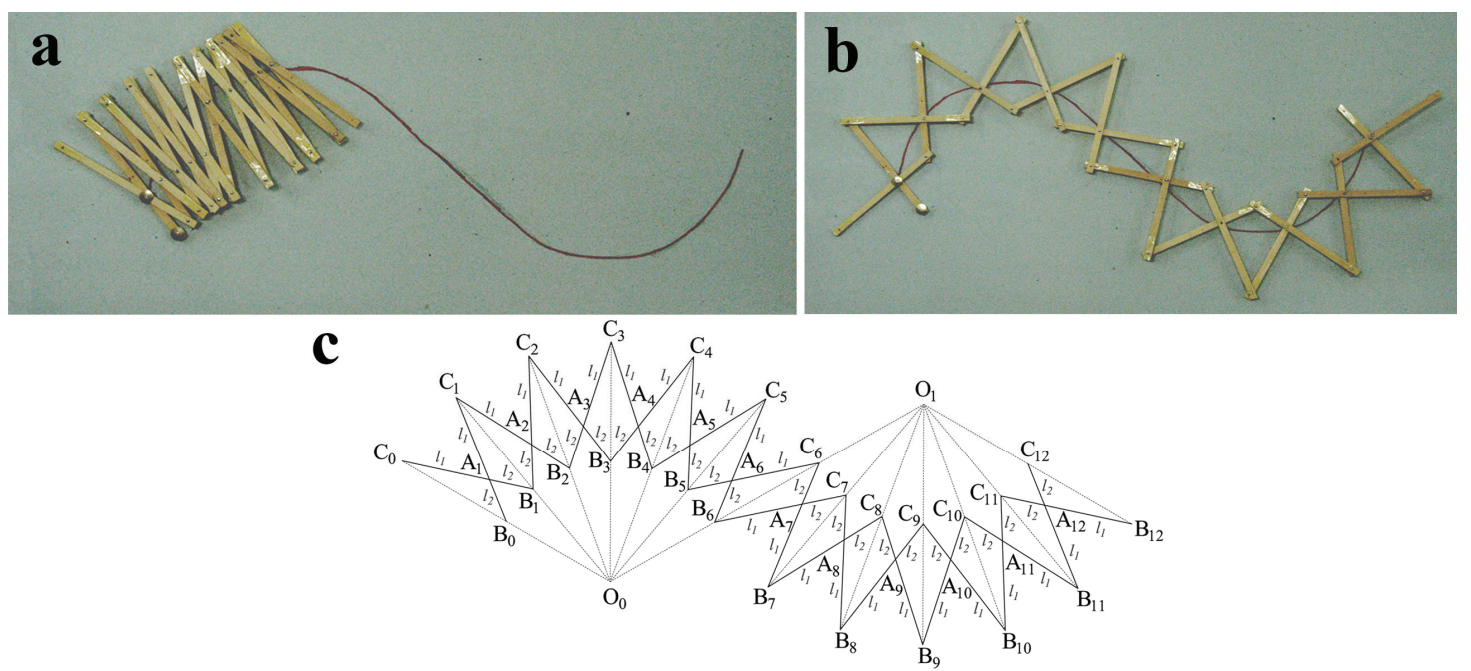

Figure 6. Path Generation of a Planar Scissor Mechanism (Maden et al, 2011)

Architects, industrial designers or engineers who focus on design of foldable/ collapsible roofs or walls, foldable furniture and building components should be familiar to the path generation in order to simplify the design process.

\subsection{Multi-loop Mechanism Synthesis}

In some conditions, common mechanisms cannot meet the expected requirements and motion paths. At these conditions, new mechanisms can be synthesized by using one of the methods for mechanism synthesis. Generation of mechanism chains (multi-loop mechanisms) is one of these methods. Main principle of this method is to join additional links to a common mechanism in order to obtain various motion paths and more complex mechanisms.

As an example to multi-loop mechanism synthesis, two different planar six bar linkages can be seen in Figure 7. Both of these two linkages have been obtained by the connection of two four-bar mechanisms. However, the linkage in Figure 7a has only one common link between these two four-bars, but the linkage in Figure 7b has two common links (Common links are represented with grey). When the equation 1 is applied to the linkage in Figure $7 \mathrm{a}$, it is seen that $J_{T}=7$ (There are two joints at points $\mathrm{A}_{1}$ and $\mathrm{B}_{1}$ ), $\lambda=3$ and $\mathrm{L}=2$, and the Mobility (M) is equal to one $(\mathrm{M}=1)$. Thus, one actuator is enough to move and stabilize the system. When the equation 1 is applied to the linkage in Figure $7 \mathrm{~b}$, it is seen that $J_{T}=8$ (There are two joints at points $\mathrm{C}_{2}$ and 
$\left.\mathrm{D}_{2}\right), \lambda=3$ and $\mathrm{L}=2$, and the Mobility (M) is equal to two $(\mathrm{M}=2)$. Thus, at least two actuators are necessary to control the linkage.

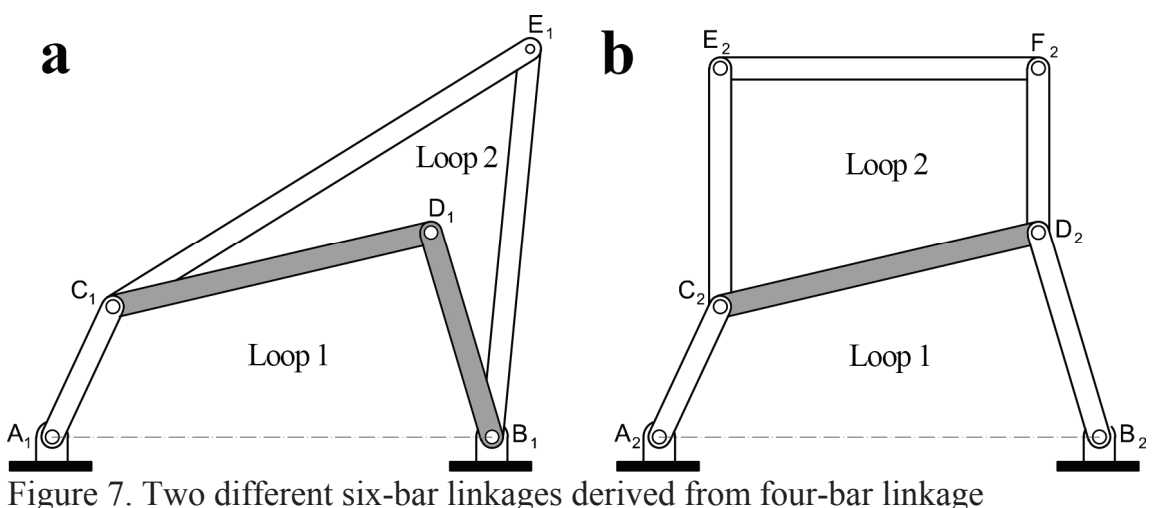

As an architectural example for multi-loop mechanism synthesis, adaptive stadium shade design of Yenal Akgün can be seen in Figure 8. The six-bar linkage in this design is obtained by the connection of two four-bar linkages. The mechanism has been synthesized analytically by using loop closure equation method (Söylemez, 2000) in Microsoft Excel Medium. By this method, all possible dimensions and geometric configurations of the mechanism can be found easily.

As it can be seen from the figure, the links which generate the linkage do not have to be linear elements. Thus, planar elements with different geometries can be used as well. The mobility of this adaptive shade is equal to one; and the mechanism can transform according to the changing climate conditions.

a

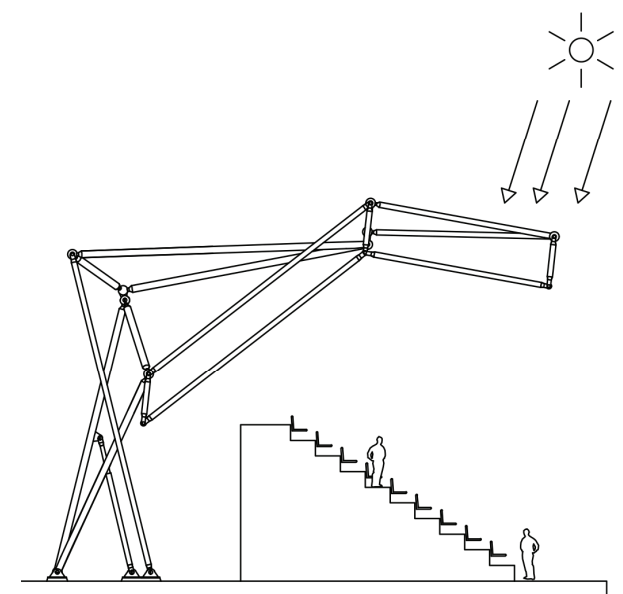

b

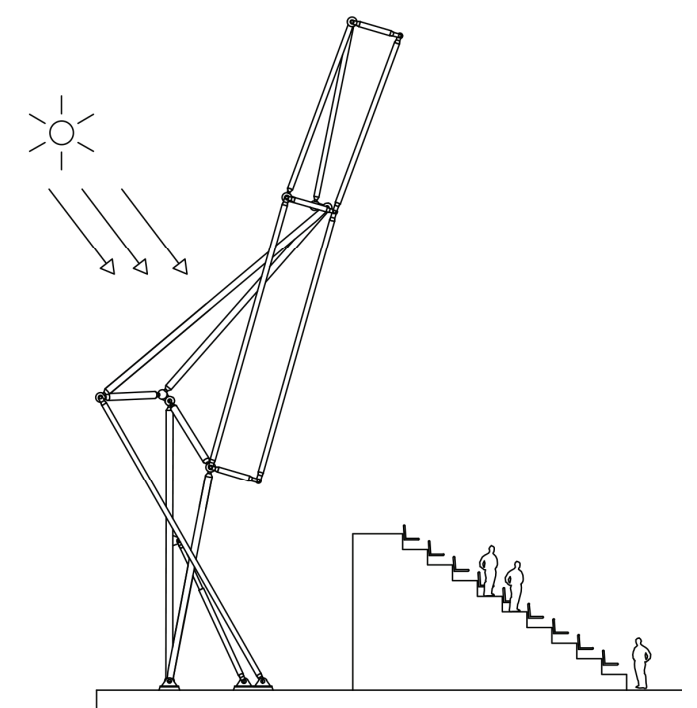

Figure 8. Adaptive Stadium Shade Design by Yenal Akgün

\section{CONCLUSIONS}

This paper has summarized the methods for kinematic synthesis of mechanisms; and tried to expose the use of these methods in design of adaptive structures. Design of adaptive structures is an interdisciplinary area between architecture, mechanical engineering and structural engineering. Only with architectural or engineering approaches, it is really difficult to develop new mechanism and also new adaptive structures. For this reason, a person who wants to be a specialist on the design of adaptive structures should be familiar both with the design part and en- 
gineering part including structural analysis, dynamics and kinematics. Beside, material science has gained importance by the rapid development of new materials.

\section{REFERENCES}

Akgün, Y., Haase W., Sobek W. 2007. Proposal for a New Scissor-Hinge Structure to Create Transformable and Adaptive Roofs. Proceedings IASS 2007 (International Association of Spatial Structures) Symposium, Venice, 3-6 September 2007.

Akgün, Y., Gantes, G., Kalochairetis, K., Kiper, G. 2010. A Novel Concept of Convertible Roofs with High Transformability Consisting of Planar Scissor-hinge Structures. Engineering Structures 32(9): 2873-2883.

Akgün, Y., Gantes, G., Sobek, W., Korkmaz, K., Kalochairetis, K. 2011. A Novel Adaptive Spatial Scissor-hinge Structural Mechanism for Convertible Roofs. Engineering Structures 33(4): 1365-1376.

Calatrava S, Santiago Calatrava, (06.11.2012), http://www.calatrava.com.

Chen, Y. 2003. Design of Structural Mechanisms, PhD thesis: University of Oxford.

Hartenberg, R.S. \& Denavit, J. 1964. Kinematic Synthesis of Linkages, New York: McGraw-Hill.

Erdman, A.G. \& Sandor, G.N. 1991. Mechanism Design: Analysis and Synthesis, Englewood Cliffs, N.J.: Prentice Hall.

Freudenstein F., Alizade R. 1975. On the Degree of Freedom of Mechanisms with Variable General Constraints. Proceedings of IFToMM IV world congress, Newcastle, 8-12 September 1975.

Korkmaz, K., Akgün, Y., Maden, F. 2012. Design of an 2-DOF 8R Linkage for Transformable Hypar Structure, Mechanics Based Design of Structures and Machines, 40: 19-32.

Maden, F., Korkmaz K., Akgün Y. 2011. A Review of Planar Scissor Structural Mechanisms: Geometric Principles and Design Methods. Architectural Science Review 54: 246-257.

McCarthy, J.M. \& Soh, G.S. 2010. Geometric Design of Linkages, New York: Springer.

Norton, R.L. 2003, Design of Machinery, New York: McGraw Hill Higher Education.

Söylemez, E. 2009. Mechanisms, Ankara: METU Press. 